\title{
A review on lignin sources and uses
}

\begin{abstract}
The efficient use of renewable resources has become a driving force for the worldwide industry aiming to improve the competitiveness. Considering the available natural raw materials, the lignin present in the lignocellulosic biomass such as trees, is the unique natural polymer that presents aromatic rings in its constitution. In this way, this review details the structure of native lignin as well as the technical lignin, including information on the characteristics that this polymer must have for the most promissory applications as feedstock for bioproducts. Approximately 50 million tons of lignin are produced worldwide annually, of which $98 \%$ to $99 \%$ is incinerated to produce energy and steam in the pulp mills. Only a small fraction of the lignin, derived mainly from the sulfite pulp mill is recovered commercially in a biorefinery concept. There are many opportunities for producing high value-added products from technical lignin, mainly considering the pulp mills growing, and environmental restrictions using non-renewable raw materials. The currently trend have shown that technical lignin sources may also be used as feedstock for phenol derived products, technical carbons, fuels, and adhesives. On the other hand, there are some technological hurdles must be overcome to make these uses feasible.
\end{abstract}

Keywords: bioproducts, biorefinery, lignocellulosic biomass, technical lignin
Volume 7 Issue 3 - 2020

Rafael Eloy de Souza,' Fernando José Borges

Gomes,' Edvá Oliveira Brito,' Roberto

Carlos Costa Lelis,' Larisse Aparecida Ribas Batalha,' Fernando Almeida Santos, ${ }^{2}$ Dalton Longue Junior ${ }^{3}$

'Forestry Products Department, Federal Rural University of Rio de Janeiro, Brazil

${ }^{2}$ Biorefinery Studies Center, State University of Rio Grande do Sul, Porto Alegre, Brazil

${ }^{3}$ Forest Engineering Course, State University of Southwest Bahia, Brazil

Correspondence: Fernando José Borges Gomes, Federal Rural University of Rio de Janeiro, BR-465, Km 7 Seropédica-Rio de Janeiro, Brazil, Zipcode 23.897-000, Tel +55 2l 268|4985,

Email fernandogomes@ufrrj.br

\section{Introduction}

The French chemist Anselme Payen was the first that treated the wood with nitric acid and sodium hydroxide, in 1838, obtaining two distinct substances, which one was called cellulose and the second one, with a high carbon content, where cellulose was soaked, being called "encrusting material". This was considered the milestone in the history of lignin. Later, in 1865 , Schulze named this "encrusting material" of lignin, from the latin Lignum, which means wood. In 1868, E. Erdmann noted that the constituents of lignin were aromatic compounds. ${ }^{1}$ Benedikt and Bamberger in 1890 demonstrated that in this lignified wood material there were also other functional groups such as methoxyl lignin is found in a large volume in the cell wall of lignocellulosic materials, such as wood, being a highly branched and amorphous biomacromolecule, and its composition can be diverse depending on the vegetal source. ${ }^{2}$ The lignin chemical structure is originated from the three hydroxycinnamyl alcohols (monolignols) called p-coumaryl alcohol, coniferyl alcohol, and sinapyl alcohol, which differ only regarding the degree of methoxylation at the $\mathrm{C} 3$ and $\mathrm{C} 5$ positions of the aromatic ring. The p-hydroxyphenyl $(\mathrm{H}$ unit) do not have any methoxyl group in the carbon $\mathrm{C} 3$ and $\mathrm{C} 5$ of the aromatic ringle, guaiacyl ( $\mathrm{G}$ unit) presents methoxyl group in the carbon $\mathrm{C}$ 3, syringyl (S unit) presents methoxyl group at carbon $\mathrm{C} 3$ and C5 (Figure 1). ${ }^{3}$

During the biological lignification process of plant species, the phenolic structure formed by the precursor alcohols is linked together, giving rise to a complex, three-dimensional structure formed by a wide variety of bonds. The main one is $\beta-\mathrm{O}-4$ ether bonds (Figure 2 ). The composition and lignin content may vary from species to species and may also be influenced by the environment. Hardwood lignin consists mainly of guaiacyl and syringyl units and low levels of p-hydroxyphenyl. Conifer lignin has higher levels of guaiacyl units and low levels of p-hydroxyphenyl. Grasses comprise guaiacyl, syringyl and p-hydroxyphenyl units. ${ }^{4}$ In all cases of biomass types these monomers are linked among them without defined repeating units. The purpose of this brief review is to summarize the different types of technical lignin that are obtained in the market and the main pathways to treat this lignin for further application to get high valueadded products.<smiles>OCC=Cc1ccc(O)cc1</smiles><smiles>COc1cc(/C=C/CO)ccc1O</smiles>

(B)<smiles>COc1cc(/C=C/CO)cc(OC)c1O</smiles>

(C)

Figure I Monolignol species: (A) p-coumaryl alcohol (H unit), (B) coniferyl alcohol (G unit), (C) sinapyl alcohol (S unit).<smiles>COc1cc(/C=C(/I)Oc2c(OC)cc(I)cc2OC)ccc1O</smiles>

Figure 2 Main linkage in a hardwood lignin: $\beta-O-4$ ether bonds. 


\section{Industrial sources of the technical lignin}

Technical lignin is originated as a bioproduct from different pretreatments or separation process where lignocellulosic biomasses are used as raw material such as for pulp or second-generation ethanol production. Depending on the used process for isolating the technical lignin its chemical properties such as composition, molecular weight, molecular structure may be impaired generating different types of lignin, with different types of applications in biorefinery platforms. They also differ in physical properties such as solubility, hydrophobicity and hydrophilicity of lignin. Because of these differences, each technical lignin must be considered individually., 5 The pulp mill is the main commercial source of lignin. The pulping processes are used to individualize the wood fibers in an extraction processes that depending on the technology applied, may occur in acid, alkaline media or using organic solvents. In all the processes the lignin is progressively broken into low molecular weight fragments. The main commercial processes are kraft, sulfite, soda, and organos solve (pilot scale). Depending on the used process a significant degradation and change the structure of the native lignin may occur, with a reduction in the amounts of aliphatic $\mathrm{OH}$ groups, $\beta-\mathrm{O}-4$, and $\beta-\beta$ bonds. It is also possible to generate degraded products such as phenolic hydroxyl groups, carboxylic acids, and carbonyl groups. ${ }^{8}$ Therefore, the structure of the technical lignin is typically dependent on the extraction method, as well as from the raw material of origin (softwood, hardwood, or grasses). ${ }^{9}$

\section{Kraft lignin}

Aiming to remove the lignin form the lignocellulosic materials, the kraft process is based on the use of a mixture of alkaline cooking chemicals, sodium hydroxide $(\mathrm{NaOH})$ and sodium sulfide $\left(\mathrm{Na}_{2} \mathrm{~S}\right)$, called white liquoras main reagent, at high temperature $\left(140-170^{\circ} \mathrm{C}\right)$. Following the delignification process, a resultant black liquor containing degraded lignin, oxidized inorganic compounds, and other organic materials which coming from the lignocellulosic material used as feedstock may is a source of the technical lignin. ${ }^{6}$ The kraft lignin obtained through the kraft pulping process, being isolated from the kraft black liquor by acidification, is highly condensed with strong ether bonds and high number of C-C bonds. Due to the chemical reagents used during the kraft pulping process, kraft lignin may have some impurities in its structure, such as sulfur. These impurities present in its structure may make its use unfeasible due to the venous nature of sulfur, or even prevent the action of catalysts. ${ }^{6}$ Kraft lignin has a large number of phenolic hydroxyls due to breakage of -aryl bonds during cooking. Oxidative conditions during the delignification process can cause formation of quinone and catechol structure, as well as the increase of carboxylic groups..$^{10,11} \mathrm{Kraft}$ lignin has a small content of ash and carbohydrate. ${ }^{5}$ It also has aliphatic thiol groups, low molecular weight and high polydispersity. ${ }^{12}$ Facing the reality of the industries report that, with the growing production of cellulose pulp, a larger amount of lignin kraft has been generated and the fact that the industries cannot consume all the produced black liquor, many studies about they are being made for conversion into high value-added products. ${ }^{13}$

\section{Lignosulfonate lignin}

It is the lignin obtained from the sulfite pulping process. In the pulping process, a mix of alkaline earth metal sulfite performs the delignification of wood, reacting with lignin to produce a water soluble sulfonated system, where the lignin is degraded by acid hydrolysis reactions. During the sulfite process, the lignin is cleaved, forming a sulfonated compound, which have a variety of functional groups providing unique colloidal properties. ${ }^{6}$ Lignosulfonates lignin are soluble in water, high molecular weight, higher ash content than kraft lignin, about $4-8 \%$. It has been used as a colloidal suspensions, stabilizers, dispersants, binders, detergents, adhesives and components of feed, surfactants, and additives for cements. $^{14}$

\section{Soda lignin}

The soda-based processes are an interesting delignification process, because they are compatible with the existing kraft pulping technology, being based on the same process conditions and principles, but they are a sulfur free process. The main chemical applied in the soda pulping process is the sodium hydroxide $(\mathrm{NaOH})$. Soda lignin is quite different from kraft lignin and lignosulfonates due to sulfurfree. Another different feature is the presence of vinyl ethers. ${ }^{6}$ Soda lignin has been applied for production of phenolic resins, dispersants, polymers synthesis. ${ }^{14}$ It also can be applied in area such as animal nutrition due to sulfur-free. ${ }^{15}$ On the other hand, the pulp mills using soda pulping process are becoming rarity, due to its low pulp yield performance. $^{16}$

\section{Organosolv lignin}

Organosolv delignification process aims to isolate the lignin from the carbohydrates in the lignocellulosic biomass using organic solvents for solubilizing the lignin. It is also interesting for many bioproducts application for being possible a sulfur free processes depending on the used solvents. ${ }^{17}$ Lignin extracted by organosolv process may have high purity, chemical reactivity, sulfur free and no toxicity. It depends on the pretreatment condition, which it is obtained. It has a homogeneous structure, almost like native lignin, low molecular weight, and polydispersity. ${ }^{6}$ Some studies show that increased severity in organosolv processes leads to a reduction in molar mass of extracted lignin by 36 to $56 \%$ compared to untreated lignin. In addition, reduction of aliphatic hydroxyl group content, increase of syringyl phenolic units and condensed phenolic structures can be observed. ${ }^{17}$ Organossolve lignin has high solubility in organic solvents and almost insoluble in water, because they are very hydrophobic. To recover organosolv lignin from the solvent, it must be precipitated, which typically involves adjust of concentration, $\mathrm{pH}$ and temperature. ${ }^{18}$

\section{Industrial lignin extraction}

Lignin is one of the most abundant renewable carbon resources. It is widely available on earth attracts much interest and can be obtained at relatively low cost. ${ }^{19}$ Lignin is the only renewable natural aromatic biopolymer that can be a candidate to replace phenol, an aromatic polymer which is from petroleum, a non-renewable resource. It is a promissory compound for being an eco-friendly substitute for aromatic chemical from petroleum. ${ }^{20}$ Native lignin has been studied for decades and the challenger around the isolation process still unclear. Isolation and purification methods may cause structural modification in different levels, depending on the process used to it. In paper and pulping process, many impurities are present in technical lignin and high condensed structures are formed. It can hinder conversion process later. ${ }^{21}$

Lignin extraction process from black liquor is the first step for the use of lignin, as it aims to recover valuable organic materials from the 
waste. The acidification process has been considered as an efficient and economical process for the isolation of lignin from black liquor. ${ }^{13}$ Among the processes of black liquor acidification for lignin extraction is the LignoBoost process. In this process, lignin is precipitated by lowering the $\mathrm{pH}$ to about 9 to 10.5 by $\mathrm{CO}_{2}$ injection. Then, the lignin precipitated is filtered. The reduction of $\mathrm{pH}$ of black liquor can result in a $\mathrm{H}_{2} \mathrm{~S}$ in the process. The precipitated lignin (slurry) is redispersed in a tank with acid filtrate which is generated at a subsequent stage of filtration. At this stage, the $\mathrm{pH}$ and temperature of the precipitation and mixing tanks are the same, but the acid concentration gradients in the wash (second filtration) stage are low. After the second stage of filtration, the precipitated lignin is crushed and dried to form lignin powder. In the LignoBoost process, the change in lignin $\mathrm{pH}$, ionic strength and solubility will occur in the slurry and not in the precipitates, which helps the properties of the precipitated lignin. ${ }^{22}$ Precipitation yield can be increased with a greater decrease in $\mathrm{pH}$, but would result in higher $\mathrm{CO}_{2}$ gas consumption, which would increase the operational cost. ${ }^{23}$ Problems related with conversion of lignin must be surmounted to achieve desirable products, including characterization of lignin and its derivatives, lignin depolymerization and product upgrading. What affect structure of lignin are biomass sources, fractionation methods and fractionation severity. ${ }^{24}$

Depolymerization condition of lignin is high reactive and it leads to an uncontrollable side reaction, including repolymerization or condensation to form new structure. ${ }^{21}$ Technical lignin obtained by soda, sulfite or kraft pulping process are usually less reactive than native lignin, because the reactive $\beta-\mathrm{O}-4$ ether linkage were replaced by recalcitrant $\mathrm{C}-\mathrm{C}$ bonds due the acid or alkali treatment. ${ }^{25}$ Complementing this information, analysis that seeks to describe and characterize this lignin are extremely significant aiming at possible applications for this material and its polymerization properties. In this review, a hardwood kraft lignin is described in Table 1, where the results came from the research projects developed by the research group using a kraft lignin extracted from an industrial black liquor.

Table I Characterization of an industrial eucalypt Kraft lignin (KL)

\begin{tabular}{lllll}
\hline & $\begin{array}{l}\text { Total } \\
\text { Lignin (\%) }\end{array}$ & $\begin{array}{l}\text { Carbohydrates } \\
(\%)\end{array}$ & $\begin{array}{l}\text { Ash } \\
(\%)\end{array}$ & Polydispersity \\
\hline $\begin{array}{l}\text { Eucalypt } \\
\text { kraft lignin }\end{array}$ & 94 & 3.4 & 2.6 & 3.1 \\
\hline
\end{tabular}

\section{Lignin activation}

Although lignin has a variety of functional groups (hydroxyl, methoxyl, carbonyl and carboxyl groups), its use is limited due to the presence of one or even two methoxyl groups in its structure, which makes it difficult to use in some products due to its low reactivity. To circumvent this limitation, several studies have been done to increase lignin's chemical reactivity by modifying its structure. These modifications consist of increasing the reactivity of hydroxyl groups or changing the nature of chemical active sites. For this, different pathways of chemical modification have been investigated to improve lignin reactive. ${ }^{26}$ For the chemical reaction of esterification, alkylation or etherification, hydroxypropylation with epoxides, urethanization via isocyanate or non-isocyanate routes, the presence of aliphatic hydroxyl groups and aromatic units facilitates the reaction. ${ }^{27}$ Phenolation is considerate one of the most promising modification methods to improve the reactivity of lignin increasing the phenolic hydroxyl group, to increase reactive sites for aromatic substitution reactions. ${ }^{28}$ In the aromatic units, chemical reaction as sulfomethylation, hydroalkylation, amination, and nitration can be an option to change chemical reactivity of lignin. Understanding lignin behavior under different chemical treatments will help to obtain a lignin with desirable properties for specific applications as demonstrated by Ganewatta (Figure 3). This has been a huge challenge for the researchers. ${ }^{28}$

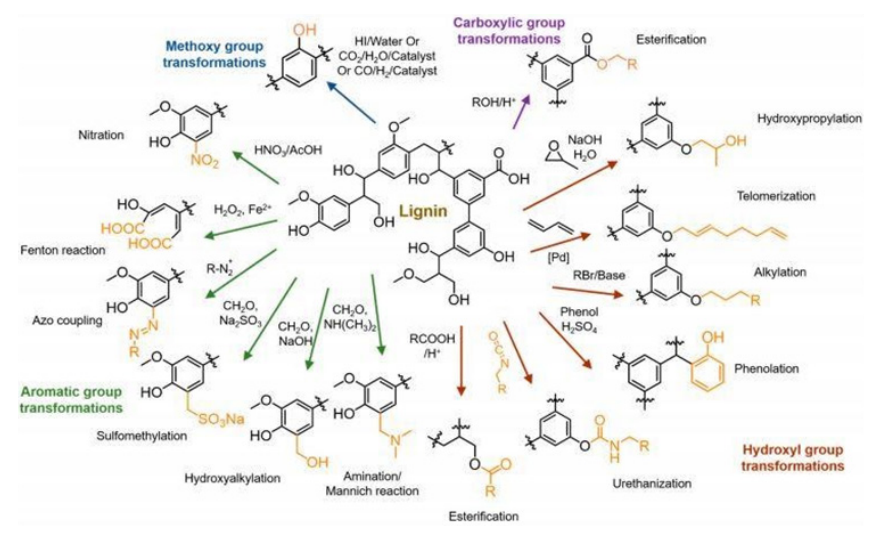

Figure 3 Different pathway for chemical treatment on lignin described by Ganewatta et al. ${ }^{27}$

\section{High value-added product from lignin}

Most of lignin produced in pulp industries as byproduct is burned for energy power. Although energy power is necessary for industry, burning lignin in order to produce heat, it is not only harmful to the environment, but also a waste of resource. ${ }^{29,30}$ Opportunities for lignin use for different purposes exist, but there are many challenges. The main valuable products potentially obtained from lignin are polymers, mixed phenols, mixed aromatic alcohols, mixed organic acids, mixed hydrocarbons, mixed quinones, mixed benzylic aldehydes, aromatics pyrolysis oil, alkyl benzenes, carbon fibers and activated carbon. ${ }^{21}$ In order to get high added-value applications for materials, lignin has been mixed with different types of polymers. However, its high complexity structure and reactivity make it difficult. Monitoring its behavior and its miscibility with other polymers is crucial to figure out its compatibility and understand the structure property relationship. ${ }^{31}$ Chemical modification of lignin is required to achieve better properties in lignin-polymer blend, such as: resins, polymers, surfactants, adsorbing agent, nucleating agent, thermal stabilizer, UV blocker, carbon fibers, super capacitor for energy storage, biomedical and pharmaceutical applications, flame retardant and biocide. ${ }^{32}$

\section{Resins}

Among most resin used in wood adhesive industry, phenolformaldehyde is commonly used due to its good performance in water and weather resisting, and high mechanical strength. Phenol and formaldehyde are both from petrol, so their prices vary with oil price. Moreover, phenol and formaldehyde are toxic and carcinogenic. ${ }^{33}$ As alternative, lignin-phenol-formaldehyde adhesives may be synthesized, since the lignin presents phenol groups in its structure. In this case, lignin is partially or completely used as substitute to phenol in the resin formulation. ${ }^{34}$ To make lignin appropriate to be used as adhesives for wood panels, several properties of lignin must be considerate, being its chemical heterogeneity is a limiting factor. ${ }^{35}$ In general, the lignin is isolated from the lignocellulosic biomass using different chemical processes, aiming to solubilize and precipitate this polymer, but during these processes the lignin structure is strongly 
affected, e.g., reducing its molecular weight and functional groups, such as phenol content. ${ }^{6,35}$ Many studies have been done to improve the synthesis lignin-phenol-formaldehyde and its applicability. Preparing lignin-phenol-formaldehyde resins substituting $40 \mathrm{wt} \%$ of phenol by pine kraft lignin, found good results in plywood improving the water absorption, thickness swelling and mechanical properties like flexural strength, elastic modulus and relative impact energy. ${ }^{36}$

\section{Technical carbon}

Due to lignin has high carbon content and aromaticity, it has been studied as precursor for production of functional materials such as activated carbon, activated carbon fiber, carbon catalyst, electrodes for electrochemical applications. It can also be used to produce a range of structural carbon materials such as carbon fibers and carbon black. ${ }^{37}$ Among all these applications, carbon fiber and activated carbon are the most valuable product for technical lignin. The study for this application has been growing rapidly. ${ }^{38}$ According to Grand View Research Inc. (2019) the USA market are expecting a boost of the demand for lightweight material, such as carbon fiber. ${ }^{39}$ Carbon fiber presents excellent mechanical properties, low weight, high thermal, able to conduct heat and electricity and shows chemical stability in the absence of oxidizing agents making this material very attractive for transportation industry, sporting goods, and large structures as turbine blades ${ }^{40,41}$ The use of carbon fiber in aircraft allow the manufacture of a lighter aircraft and high strength as well as it can be used to produce multifunctional structural parts capable of de-icing or protect aircraft wings from thunder due to its property of good electrical conductivity. ${ }^{42}$ Currently, about $90 \%$ of commercial carbon fiber production, polyacrylonitrile is used as precursor. Polyacrylonitrile is a synthetic polymer derived from petroleum that represents half the cost of producing carbon fiber, making this a cost-limitation for carbon fiber applications..$^{43,44}$

Lignin has an attractive attention to replace polyacrylonitrile in the carbon fiber manufacturing due to its high carbon content, as well as its bioavailability and low cost. Another advantage is the high carbon yield during carbonization and the absence of toxic gases during the carbonization of polyacrylonitrile. ${ }^{45}$ The major challenge for this use is due to the intrinsic heterogeneity characteristic of lignin such as different molecular weight, diverse chemical linkages, and many functional groups making the lignin a heterogeneous polymer. It contributes to the poor mechanical performance of lignin-based carbon fiber. ${ }^{46}$

\section{Lignin for chemical}

We can use lignin as source to replace petrochemical derived aromatic monomers. Depolymerization process is a good method to obtain low-molecular-weight compound from lignin. ${ }^{46}$ As example of depolymerization techniques we can cite: pyrolysis, ${ }^{47}$ catalytic hydrogenolysis, solvent depolymerization, alkaline oxidation, supercritical water, alkaline hydrolysis. ${ }^{48}$ Among the depolymerization technique, the feasibility of lignin depolymerization process depends of the technique and chemical composition of technical lignin. ${ }^{49}$ Compound such as vanillin, divanillin, ferulic acid, hydroxybenzaldehyde, syringaldehyde and guaiacol can be obtained from the depolymerization process. ${ }^{27}$

Among these monomers, vanillin has gained significant interest for its use as a precursor to polymer synthesis. It is produced from petrochemical source, but kraft lignin source can be more environment-friendly and potential economic than from guaiacol. For this, the main challenge is to purify the kraft lignin for using in vanillin products, since the vanillin can be sold as natural aroma just if the base material and the process are natural. Hence, numerous strategies are being developed to use biotechnology to produce vanillin using kraft lignin. ${ }^{50}$

\section{Lignosulfonates}

Kraft lignin is also being tested as coagulant and dispersants. Due to low solubility at neutral $\mathrm{pH}$ and limited charge density, kraft lignin has received chemical treatment to improve it. ${ }^{51}$ Sulfonation process has been applied to lignin so it can be used as coagulant or dispersant. Treat kraft lignin by phenolation followed sulfonation of sodium sulfite and sulfuric acid. In their experiment lignin presented good performance as coagulant for dye removal from their simulated solution. However, sodium sulfite lignin required higher dosage, because it has lower charge density than sulfuric acid lignin. ${ }^{52}$ In a study treating lignin chemically with sulfuric acid and sodium sulfite, it improved the solubility across the range of $\mathrm{pH}$ when compared with non-treat lignin. According to its results, the authors found favorable results to use modified lignin as dispersant..$^{53}$

\section{Conclusion}

There are many opportunities for producing high value-added products from technical lignin, mainly considering the pulp mills growing, and environmental restrictions using non-renewable raw materials. On the other hand, there are still many challenges to be overcome in order to make lignin an industrially applicable feedstock.

\section{Acknowledgments}

Financial support provided by the Brazilian Agencies CAPES, $\mathrm{CNPq}$ and FAPERJ are gratefully acknowledged.

\section{Conflicts of interest}

There is no conflict of interest in publishing the article.

\section{Funding}

None.

\section{References}

1. Mahmood Z, Yameen M, Jahangeer M, et al. Lignin as Natural Antioxidant Capacity. In: Poletto M, editor. Lignin - Trends and Applications, InTech. 2018. p.181-205.

2. Sjostrom E. Wood chemistry: fundamentals and applications. San Diego, CA; 1993. p. 1-250.

3. Erdtman H. Lignins: Occurrence, formation, structure and reactions. In: KV Sarkanen, CH Ludwig, editors. John Wiley \& Sons, Inc., New York. Journal of Polymer Science Part B: Polymer Letters. 1972;10(3):228230 .

4. Upton BM, Kasko AM. Strategies for the conversion of lignin to highvalue polymeric materials: review and perspective. Chemical reviews. 2016;116(4):2275-2306.

5. Vishtal AG, Kraslawski A. Challenges in industrial applications of technical lignins. Bio Resources. 2011;6(3):3547-3568.

6. Kumar A, Kumar J, Bhaskar T. Utilization of lignin: A sustainable and eco-friendly approach. Journal of the Energy Institute. 2020;93(1):235271 . 
7. Doherty WO, Mousavioun P, Fellows CM. Value-adding to cellulosic ethanol: Lignin polymers. Industrial crops and products. 2011;33(2):259276.

8. Capanema EA, Balakshin M. Plantrose lignins: a new type of technical lignins. In Proceedings of 18th ISWFPC-International Symposium on Wood, Fiber and Pulping Chemistry. 2015. p. 120-123.

9. Capanema EA, Balakshin MY. U.S. Patent No. 10,059,730. Washington, DC: U.S. Patent and Trademark Office. 2018.

10. Chakar FS, Ragauskas AJ. Review of current and future softwood kraft lignin process chemistry. Industrial crops and products. 2004;20(2):131141.

11. Asgari F, Argyropoulos DS. Fundamentals of oxygen delignification. Part II. Functional group formation/elimination in residual kraft lignin. Canadian journal of chemistry. 1998;76(11):1606-1615.

12. Lin X, Zhou M, Wang S, et al. Synthesis, structure, and dispersion property of a novel lignin-based polyoxyethylene ether from kraft lignin and poly (ethylene glycol). ACS Sustainable Chemistry \& Engineering. 2014;2(7):1902-1909.

13. Luong ND, Binh NT, KimT, et al. An eco-friendly and efficient route of lignin extraction from black liquor and a lignin-based copolyester synthesis. Polymer bulletin. 2012;68(3):879-890.

14. Jablonský M, Kočiš J, Ház A, et al. The approach the isolation of lignins and its characterization. Power Engineering. 2014;27-32.

15. Baurhoo B, Ruiz-Feria CA, Zhao X. et al. Purified lignin: Nutritional and health impacts on farm animals - A review. Animal Feed Science and Technology. 2008;144(3-4):175-184.

16. de Almeida DP, Gomide JL. Anthraquinone and surfactant effect on soda pulping. O Papel. 2013;74:53-56.

17. Yáñez-SM, Matsuhiro B, Nuñez C, et al. Physicochemical characterization of ethanol organosolv lignin (EOL) from Eucalyptus globulus: Effect of extraction conditions on the molecular structure. Polymer degradation and stability. 2014;110:84-194.

18. Vázquez G, Antorrena G, González J, et al. The influence of pulping conditions on the structure of acetosolv eucalyptus lignins. Journal of wood chemistry and technology. 1997;17(1-2):147-162.

19. Alonso DM, Wettstein SG, Dumesic JA. Bimetallic catalysts for upgrading of biomass to fuels and chemicals. Chemical Society Reviews. 2012;41(24):8075-8098.

20. Isikgor FH, Becer CR. Lignocellulosic biomass: a sustainable platform for the production of bio-based chemicals and polymers. Polymer Chemistry. 2015;6(25):4497-4559.

21. Wang H, Pu Y, Ragauskas A, et al. From lignin to valuable productsstrategies, challenges, and prospects. Bioresource Technology. 2019;271:449-461.

22. Fatehi Pedram, Jiachuan Chen. Extraction of technical lignins from pulping spent liquors, challenges and opportunities." Production of biofuels and chemicals from lignin. Springer, Singapore; 2016:35-54.

23. Öhman F, Wallmo H, Theliander H. Precipitation and filtration of lignin from black liquor of different origin. Nordic Pulp \& Paper Research Journal. 2007;22(2):188-193.

24. Kim KH, Dutta T, Sun J, et al. Biomass pretreatment using deep eutectic solvents from lignin derived phenols. Green chemistry. 2018;20(4):809815.

25. Li C, Zhao X, Wang A, et al. Catalytic transformation of lignin for the production of chemicals and fuels. Chemical reviews. 2015;115(21):11559-11624.:
26. Laurichesse S, Avérous L. Chemical modification of lignins: Towards biobased polymers. Progress in polymer science. 2014;39(7):1266-1290.

27. Ganewatta MS, Lokupitiya HN, Tang C. et al. Lignin Biopolymers in the Age of Controlled Polymerization. Polymers. 2019;11(7):1176.

28. Jiang X, Liu J, Du X, et al. Phenolation to improve lignin reactivity toward thermosets application. ACS Sustainable Chemistry \& Engineering. 2018;6(4):5504-5512.

29. Bruijnincx PC, Weckhuysen BM. Lignin up for break-down. Nature chemistry. 2014;6(12):1035-1036.

30. Simão L, Hotza D, Raupp-Pereira F, et al. Wastes from pulp and paper mills-a review of generation and recycling alternatives. Cerâmica. 2018;64(371):443-453.

31. Sen S, Patil S, Argyropoulos DS. Thermal properties of lignin in copolymers, blends, and composites: a review. Green Chemistry. 2015;17(11):4862-4887.

32. Collins MN, Nechifor M, Tanasă F, et al. Valorization of lignin in polymer and composite systems for advanced engineering applications-a review. International journal of biological macromolecules. 2019.

33. Foyer G, Chanfi BH, Virieux D, et al. Aromatic dialdehyde precursors from lignin derivatives for the synthesis of formaldehyde-free and high char yield phenolic resins. European Polymer Journal. 2016;77:65-74.

34. Hussin MH, Zhang HH, Aziz NA, et al. Preparation of environmental friendly phenol-formaldehyde wood adhesive modified with kenaf lignin. Beni-Suef University journal of basic and applied sciences. 2017;6(4):409-418.

35. Mahendran AR, Wuzella G, Aust N, et al. Processing and characterization of natural fibre reinforced composites using lignin phenolic binder. Polymers and Polymer Composites. 2013;21(4):199-206.

36. Ghorbani M, Mahendran AR, van Herwijnen HW, et al. Paper based laminates produced with kraft lignin-rich phenol-formaldehyde resoles meet requirements for outdoor usage. European journal of wood and wood products. 2018;76(2):481-487.

37. Puziy AM, Poddubnaya OI, Sevastyanova O. Carbon materials from technical lignins: Recent advances. In Lignin Chemistry. Springer, Cham; 2020. p. 95-128.

38. Gosselink RJA. Lignin as a Renewable Aromatic Resource for the Chemical Industry, PhD thesis. Wageningen University, Wageningen, NL; 2011.

39. Grand view research Inc. Lignin Market Analysis By Product (LignoSulphonates, Kraft Lignin, Organosolv Lignin) By Application (Macromolecules, Aromatics), By Region (APAC, MEA, North America, Europe), and Segment Forecasts. 2019.

40. Witten E, Kraus T, Kühnel M. Composites Market Report 2015-Market developments, trends, outlook and challenges. Carbon Composites AVK. 2015.

41. Rajak DK, Pagar DD, Menezes PL, et al. Fiber-Reinforced Polymer Composites: Manufacturing, Properties, and Applications. Polymers. 2019;11(10):1667.

42. Forintos N, Czigany T. Multifunctional application of carbon fiber reinforced polymer composites: electrical properties of the reinforcing carbon fibers-a short review. Composites Part B: Engineering. 2019;162:331-343.

43. Baker DA, Rials TG. Recent advances in low-cost carbon fiber manufacture from lignin. Journal of Applied Polymer Science. 2013;130(2):713-728.

44. Frank E, Steudle LM, Ingildeev D, et al. Carbon fibers: precursor systems, processing, structure, and properties. Angewandte Chemie International Edition. 2014;53(21):5262-5298. 
45. Huang X. Fabrication and properties of carbon fibers. Materials. 2009;2(4):2369-2403.

46. Li Q, Xie S, Serem WK, et al. Quality carbon fibers from fractionated lignin. Green Chemistry. 2017;19(7):1628-1634.

47. Sun Z, Fridrich B, de Santi A, et al. Bright side of lignin depolymerization: toward new platform chemicals. Chemical reviews. 2018;118(2):614678.

48. Pandey MP, Kim CS. Lignin depolymerization and conversion: a review of thermochemical methods. Chemical Engineering \& Technology. $2011 ; 34(1) ; 29-41$.

49. Ponnusamy VK, Nguyen DD, Dharmaraja J, et al. A review on lignin structure, pretreatments, fermentation reactions and biorefinery potential. Bioresurce technology. 2019;271:462-472.
50. Fache M, Boutevin B, Caillol S. Vanillin, a key-intermediate of biobased polymers. European polymer journal. 2015;68:488-502.

51. Coughlin JE, Reisch A, Markarian MZ, et al. Sulfonation of polystyrene: Toward the "ideal" polyelectrolyte. Journal of Polymer Science Part A: Polymer Chemistry. 2013;51(11):2416-2424.

52. Gao W, Inwood JP, Fatehi P. Sulfonation of phenolated kraft lignin to produce water soluble products. Journal of Wood Chemistry and Technology. 2019;39(4):225-241.

53. Inwood JP, Pakzad L, Fatehi P. Production of sulfur containing kraft lignin products. Bio Resources. 2018;13(1):53-70. 